\title{
Health-related quality of life (HRQL) for individuals with self-reported chronic physical and/or mental health conditions: panel survey of an adult sample in the United States
}

Martha Bayliss, Regina Rendas-Baum, Michelle K White, Mark Maruish, Jakob Bjorner and Sandra L Tunis*

\begin{abstract}
Background: In the US, approximately 53\% of adults have at least one chronic condition. Comorbid physical and mental health conditions often have an incremental negative impact on health-related quality of life (HRQL). Primary study objectives were to quantify the impact on HRQL of a) $\geq 1$ physical condition , b) $\geq 1$ comorbid mental health conditions added to a physical one, c) $\geq 1$ mental health condition, and d) $\geq 1$ comorbid physical conditions added to at least one related to mental health. Decrements were based on a "Healthy" reference group reporting no chronic conditions.
\end{abstract}

Methods: Participants were sampled $(n=3877)$ from the US adult population as part of a 2009 normative survey. Demographics, number/ type of chronic conditions, and HRQL data were self-reported. HRQL was defined through SF-36v2 ${ }^{\circledR}$ Physical Component Summary (PCS) scores and Mental Component Summary (MCS) scores. Participant "morbidity" groupings included Healthy; Physical Health Condition only, Mental Health Condition only, and Physical and Mental Health (Comorbid). PCS and MCS scores were also analyzed by physical disease clusters (e.g., cardiovascular, gastrointestinal). Multivariate regression models were used for all analyses.

Results: $81 \%$ of participants were Caucasian; $9 \%$ African American. Males and females were about equally represented; $63 \%$ were $\geq 45$ years old. The average number of reported chronic conditions was $2.4(\mathrm{SD}=2.4)$. Relative to the Healthy group, the Physical Condition group scored 6.4 (males) and 7.5 (females) points lower on PCS. The addition of a comorbid mental health condition resulted in a total reduction of 11 points in PCS and 15 points in MCS. Compared to the Healthy group, $\geq 1$ mental health conditions was associated with MCS decrements of 11-12 points. A physical comorbidity led to additional decrements of 3-4 points for MCS, with a total of 15 points. Incremental HRQL burden defined by both MCS and PCS scores was relatively similar across the 5 defined physical disease clusters.

Conclusion: Results provide quantitative information for US adults on specific PCS and MCS score decrements associated with a comorbid condition related to mental health, as well as a comorbid condition related to physical health.

\footnotetext{
*Correspondence: stunis@qualitymetric.com

QualityMetric, Inc., part of Optumlnsight, 24 Albion Road, Building 400, Lincoln, RI 02865, USA
} 


\section{Background}

\section{Chronic conditions in the United States}

The humanistic and economic burden of chronic health conditions has been well-documented in the United States (US) and elsewhere. In the US, approximately half (53\%) of the adult population has at least one chronic condition related to physical or mental health [1]. Moreover, $7 \%$ of adults $45-54$ years of age, and $37 \%$ of those 75 years or older report having three or more [2]. Although most chronic conditions can be controlled through treatment and long-term management, they are the primary reason for seeking medical care and the leading contributors to disability and mortality [3].

Certain chronic conditions related to physical health are of particular concern due to their comparatively high prevalence, as well as their link to additional morbidities and negative effects on daily functioning. For example, the US prevalence of diabetes for those at least 20 years of age is approximately $11 \%$ [4], and has been linked to a variety of cardiovascular and other types of complications $[4,5]$. The overall prevalence of coronary heart disease (CHD) for US adults is 6-7\%, and remains a leading cause of morbidity and mortality [6]. Finally, about onethird of adults over age 65 experience chronic symptomatic osteoarthritis, the leading cause of disability in older adults [7].

Several chronic conditions related to mental health are also comparatively prevalent in the US adult population. The lifetime and 12-month prevalence of any anxiety disorder is $29 \%$ and $18 \%$, respectively. The prevalence of major depression is $17 \%$ (lifetime) and 7\% (12-month) $[8,9]$.

\section{Physical and mental health comorbid chronic conditions}

A large body of research documents the co-occurrence of a specific chronic physical condition (e.g., congestive heart failure ( $\mathrm{CHF})$, diabetes) and one related to mental health such as depression or anxiety [10-13]. Certain chronic conditions frequently precipitate and/or exacerbate depressive symptoms [14,15]. Conversely, depression can lead to- or worsen conditions in the physical domain such as asthma, cardiovascular disease, and diabetes $[15,16]$. Comorbid physical and mental health conditions can show a combined negative impact on disease-related outcomes $[7,15]$.

To illustrate, among participants with type 2 diabetes $(\mathrm{n}=249)$ in a UK study, those with anxiety and/or depression reported significantly more days off work, as well as decreased adherence to diabetes medication ( $\mathrm{p}<.05$ for both outcomes) [17]. In another study of veterans with type 2 diabetes, the presence of comorbid depression and/or alcohol/drug abuse had a significant and negative impact on diabetes-specific outcomes, as well as all-cause mortality [18].

\section{Chronic conditions and Health-Related Quality of Life (HRQL)}

Studies assessing the burden of chronic physical and mental health conditions increasingly include the negative impact on an individual's health-related quality of life (HRQL), commonly defined as everyday functioning and well-being [19-22]. One recent study of US adults ( $n=4833$ ) retrospectively compared self-reported burden of chronic conditions related to mental health, to those of three related to physical health (back/neck problems, hypertension, diabetes) [23]. Burden was defined by the number of days in the previous 30 when a person indicates that activities are limited due to physical or mental health difficulties [24-26].

Controlling for demographic variables, adults with mental health conditions reported 6.8 fewer healthy days/ month compared to those without these conditions $(\mathrm{p}<0.001)$. Among adults with one of the 3 physical conditions, the mean number of additional unhealthy days (versus those with none of these physical conditions) ranged from 1.0 (for hypertension, n.s.) to 3.6 (back and neck problems, p $<0.001$ ). When compared directly, mental health conditions were associated with significantly lower HRQL than were any of the 3 related to physical health ( $\mathrm{p}=0.002$ to 0.053 ).

Aggregating different chronic conditions, Rothrock et al. [27] examined the association of number of conditions and HRQL. Study participants $(\mathrm{n}=21,133)$ reported the presence or absence of 24 possible chronic health conditions, and whether or not their activity was limited by each. Across all 5 health-status domains assessed (physical function, fatigue, pain, emotional distress, and social function), the presence of a chronic condition was associated with poorer scores relative to no chronic condition. For those with $\geq 2$ morbidities, decrements in HRQL were significantly more pronounced when compared to those with no condition, or to those with a single condition $(\mathrm{p}<.0001)$.

Finally, a large recently published retrospective study of US adults [24] was designed to examine HRQL based on both number and type of chronic conditions. From Behavioral Risk Factor Surveillance System (BRFSS) 2007 data [28], $57 \%$ of the sample $(n=430,912)$ reported at least 1 chronic condition; with arthritis, obesity, and hypertension the most prevalent.

With adjustments for relevant variables (age, sex, race/ ethnicity, education, income, employment, marital status, health insurance, smoking, alcohol consumption, physical activity), those with $\geq 3$ conditions were more likely to report fair or poor health (for general health, mental distress, physical distress, activity limitations) compared to those reporting 1 or 2 chronic conditions. Compared to other diseases, cardiovascular conditions or diabetes had the largest impact on HRQL. Individuals 
with one of these two conditions were 7-8 times more likely (than were those with no conditions) to report poor or fair HRQL.

\section{Use of the SF-36 health survey in assessing HRQL impact of chronic conditions}

Many studies [22,29-32] have examined the association of chronic conditions and HRQL as defined by the Physical Component Summary (PCS) and the Mental Component Summary (MCS) scores of the widely-used SF-36 ${ }^{\circledR}$ Health Survey [33-35]. The domains of physical functioning (PF), role-physical (RP), bodily pain (BP), and general health $(\mathrm{GH})$ correlate most highly with the PCS, while the mental health $(\mathrm{MH})$, role-emotional (RE), vitality (VT) and social functioning (SF) correlate most highly with the MCS.

A systematic review of published studies (1990-2003) examining multi-morbidity and HRQL among individuals in primary care treatment settings showed that 22 of the 30 studies examined used the SF-36 as the main tool to assess HRQL [30]. Although use of a standard tool greatly enhanced the comparability of results across studies, a common methodological limitation noted in the review was the failure to evaluate the effect of mental health comorbidity.

A more recent study [29] reported that the negative impact of mental health conditions on PCS scores was greater than the negative impact of various physical health conditions on MCS. Given the relatively high prevalence of several mental health conditions in the US, the cumulative impact of physical and mental health comorbidity on both Physical and Mental Health domains of HRQL warrants further examination.

\section{Study objectives}

The goal of this study was to examine the burden of self-reported chronic physical and/or mental health conditions on HRQL. Primary objectives were to quantify the HRQL impact (compared to a "Healthy" group reporting no chronic conditions) of a) at least 1 physical condition, , b) 1 or more comorbid mental health conditions added to a physical one, c) at least 1 mental health condition, and d) 1 or more comorbid physical conditions added to a mental health one. A secondary objective was to examine the extent to which patterns of HRQL burden noted overall were similar across specific organ-system domains or Physical "Disease Clusters".

\section{Methods}

\section{Participant sample and data collection}

The current study was part of a QualityMetric 2009 Norming Study conducted in 2009 [35]. Study participants were sampled from the US general population of (non-institutionalized) adults aged 18 or older who were fluent in English. They were recruited through the Knowledge Panel ${ }^{\circledR}$ maintained by Knowledge Networks [36] (now GfK Custom Research, LLC), using a validated method of probability sampling of residential addresses covering approximately $97 \%$ of US households. All data were based on self-report and were collected via the Internet between June and October of 2009. Demographic information obtained included age, gender, race, education, and employment and marital status.

\section{Assessment of chronic conditions}

Using a chronic condition checklist, participants reported whether they had ever been told by a doctor or other health care professional that they had any of 26 possible chronic conditions. The 23 chronic physical conditions included those related to the cardiovascular, endocrine, respiratory, musculoskeletal, and gastrointestinal domains, as well as other conditions such as migraine headaches, and cancer. The three chronic mental health conditions in the checklist were clinical depression, anxiety, and an alcohol or other substance use disorder.

\section{Assessment of Health-Related Quality of Life (HRQL)}

Participants completed the SF-36v2 ${ }^{\circledR}$ Health Survey (SF$36 \mathrm{v} 2$ ), a widely-used generic measure of HRQL [35]. The SF-36v2 has been extensively validated, and has been used to assess and compare disease burden, as well as treatment effectiveness in a very large number of chronic health conditions, including those related to both physical and mental health $[29,30]$. The eight domains derived from 36 item responses were aggregated into the Physical Component Summary (PCS) and the Mental Component Summary (MCS) scores.

Based on a large sample of adults in the US, the two extensively documented component summary measures are each scored on the $T$-score metric, with a mean of 50.0 (and a standard deviation of 10.0). As with the prior version of the SF-36, one important aspect of the SF-36v2 construct validation procedures included knowngroups comparisons $[34,35]$. These analyses demonstrated the ability of the PCS and MCS measures to discriminate groups of individuals with only a physical or a mental health condition from a "healthy" group and from groups with comorbid (physical and mental health) conditions [35].

\section{Statistical analyses}

Based on checklist responses, four "morbidity" groups were created for comparisons relevant to the primary study objectives:

1. Healthy: no reported chronic conditions related to physical or mental health. 
2. Physical Health Condition: 1 or more chronic physical conditions, but none related to mental health.

3. Mental Health Condition: 1 or more chronic mental health conditions, but none related to physical health.

4. Physical and Mental Health (Comorbid) Condition: one or more chronic physical conditions and one or more related to mental health.

The mean PCS and MCS scores of participants in the a) Physical Health Condition group, and b) Mental Health Condition group were separately compared to scores of those in the Healthy group to evaluate the HRQL impact of having one or more chronic physical conditions or one or more related to mental health. Further, a comparison of the a) Physical Health Condition group and b) Physical and Mental Health Condition (Comorbidity) group was made to evaluate the impact of $\geq 1$ mental health conditions in addition to $\mathrm{a} \geq 1$ physical conditions. Finally, a comparison of the a) Mental Health Condition group and b) Physical and Mental Health Condition (Comorbidity) group was conducted to evaluate the HRQL impact of $\geq 1$ physical conditions in addition to a having $\geq 1$ mental health conditions.

Mean PCS and MCS scores for each of the defined "morbidity" groups were estimated using multivariate regression models with group membership, age, and an interaction term between group and age as independent variables. Mean PCS and MCS values were estimated by setting age at the mean of the entire sample of Normative Study participants (51 years). This was done to ensure that PCS and MCS were estimated at the same value of age for all morbidity groups and for both males and females, so as to ensure a comparability of results. Models were run to obtain comparisons separately for males and females.

Secondary objective: analyses by physical disease cluster To address the secondary objective, a subset of physical conditions was categorized into one of five physical disease clusters listed below. Participants were then grouped by Disease Cluster. This clustering approach was used to overcome cumbersome analytics and sample requirements involved with studying individual conditions $[29,37,38]$.

1. Cardiovascular Cluster: heart attack in past year; congestive heart failure (CHF); angina or coronary artery disease; other heart conditions

2. Gastrointestinal Cluster: liver disease; kidney disease; stomach disease; irritable bowel syndrome (IBS)

3. Endocrine Cluster: type 1 diabetes, type 2 diabetes
4. Musculoskeletal Cluster: rheumatoid arthritis (RA); osteoarthritis; degenerative arthritis; osteoporosis

5. Respiratory Cluster: chronic obstructive pulmonary disease (COPD)

The incremental PCS burden of a comorbid mental health condition was evaluated by comparing, within each disease cluster, the mean PCS scores of participants with no mental health conditions to the mean PCS scores of those who also had at least 1 mental health condition. Conversely, the incremental MCS burden of a physical comorbidity was evaluated by comparing the mean MCS scores of participants reporting $\geq 1$ mental health condition but no physical health conditions, to the mean MCS scores of those reporting $\geq 1$ mental health conditions and $\geq 1$ condition from each "Physical Disease Cluster" specified above.

\section{Results}

\section{Participant characteristics}

As shown in Table 1, the majority of the 3,877 study participants were Caucasian (81\%), and 9\% were African American. Males (49\%) and females (51\%) were represented almost equally. Approximately equal percentages reported their education level as high school completion $(30 \%)$; some college $(31 \%)$; or a bachelor's degree or higher (31\%). More than half were working (53\%) and almost one fourth $(24 \%)$ reported being retired. Fifty-eight percent reported being married or living with a partner, $15 \%$ being separated or divorced, and 20\% never having been married.

As also shown in Table 1, 63\% of those in the study were at least 45 years of age and 36\% at least age 60 . This distribution is reflective of the intentional oversampling of individuals age 65 and older for the 2009 Normative Survey. However, this oversampling did not bias the regression results as all regression analyses were conducted using weights that adjusted the age and gender distributions of the study sample to the age and gender distributions of the 2009 US general population. A total of 1,505 participants were included in the Healthy group; 1,522 in the Physical Health Condition; 236 in the Mental Health Condition; and 614 in the Physical and Mental Health Condition (Comorbidity) groups.

\section{Reporting frequency of specific conditions}

Overall, the average number of chronic conditions reported was $2.6(\mathrm{SD}=2.5)$. The five most commonly reported were anxiety $(n=629,16 \%)$, osteoarthritis and degenerative arthritis $(n=583,15 \%)$, diabetes $(n=580$, $15 \%)$, migraine $(\mathrm{n}=574,15 \%)$ and depression $(\mathrm{n}=507$, 
Table 1 Sample characteristics by "Morbidity Group"

\begin{tabular}{|c|c|c|c|c|c|}
\hline & Healthy $(1,505)$ & Physical $(1,522)$ & Mental (236) & Physical \& Mental (614) & Total sample $(3,877)$ \\
\hline \multicolumn{6}{|l|}{ Mean (SD) } \\
\hline Age & $44.5(16.2)$ & $58.9(15.8)$ & $40.0(14.4)$ & $51.4(14.5)$ & $51.0(17.2)$ \\
\hline Selected physical conditions & $0.0(0)$ & $1.9(1.2)$ & $0.0(0)$ & $2.6(1.7)$ & $1.2(1.5)$ \\
\hline Total number of conditions & $0.7(0.8)$ & $3.5(2.0)$ & $1.6(1.2)$ & $5.5(2.7)$ & $2.6(2.5)$ \\
\hline \multicolumn{6}{|c|}{ Percent } \\
\hline \multicolumn{6}{|l|}{ Age } \\
\hline $18-29$ & 23.7 & 6.6 & 31.8 & 9.9 & 15.3 \\
\hline $30-44$ & 29.1 & 12.6 & 32.2 & 22.8 & 21.8 \\
\hline $45-59$ & 26.0 & 25.4 & 25.4 & 36.0 & 27.3 \\
\hline $60+$ & 21.2 & 55.5 & 10.6 & 31.3 & 35.6 \\
\hline Male gender & 56.8 & 47.9 & 41.9 & 37.5 & 49.3 \\
\hline \multicolumn{6}{|l|}{ Race/ethnicity } \\
\hline White & 78.4 & 82.8 & 83.5 & 80.5 & 80.8 \\
\hline Black or African American & 10.6 & 8.9 & 5.9 & 8.1 & 9.3 \\
\hline American Indian or Alaskan Native & 0.5 & 0.9 & 0.8 & 1.6 & 0.9 \\
\hline Asian & 2.3 & 1.2 & 1.7 & 0.5 & 1.5 \\
\hline Other & 4.1 & 3.2 & 5.5 & 6.0 & 4.1 \\
\hline Missing & 4.1 & 3.0 & 2.5 & 3.3 & 3.4 \\
\hline \multicolumn{6}{|l|}{ Education } \\
\hline Less than high school & 6.5 & 9.3 & 7.6 & 10.7 & 8.3 \\
\hline High school & 28.7 & 32.3 & 27.5 & 29.8 & 30.2 \\
\hline Some college & 30.6 & 29.7 & 32.2 & 34.7 & 31.0 \\
\hline Bachelor's degree or higher & 34.2 & 28.8 & 32.6 & 24.8 & 30.5 \\
\hline \multicolumn{6}{|l|}{ Current employment status } \\
\hline Working & 66.9 & 42.8 & 64.4 & 40.4 & 53.1 \\
\hline Not working (looking) & 9.8 & 3.8 & 15.3 & 8.5 & 7.6 \\
\hline Retired & 13.3 & 40.1 & 6.8 & 19.1 & 24.3 \\
\hline Not Working (not looking) & 10.0 & 13.3 & 13.6 & 32.1 & 15.0 \\
\hline \multicolumn{6}{|l|}{ Marital Status } \\
\hline Married/Living with partner & 60.1 & 61.2 & 51.7 & 49.7 & 58.4 \\
\hline Widowed & 3.2 & 10.4 & 0.8 & 7.0 & 6.5 \\
\hline Separated/Divorced & 11.4 & 14.4 & 13.1 & 24.1 & 14.7 \\
\hline Never married & 25.2 & 14.0 & 34.3 & 19.2 & 20.4 \\
\hline
\end{tabular}

13\%). Table 2 provides a list of chronic conditions included in the present study, with corresponding frequency of self-report for both males and females.

Considering all conditions included in the analysis, women reported having more conditions than did men $($ mean $=2.8[\mathrm{SD}=2.7]$ for women and mean $=2.0$ $[\mathrm{SD}=2.1]$ for men). As expected, there were differences between males and females in the ordering of conditions by frequency with which they were reported. Migraine was more common in women (21.9\%) than men $(7.5 \%)$, as was anxiety $(20.2 \%$ for women versus
$12.2 \%$ for men), depression (17\% for women versus $9 \%$ for men) and osteoporosis (10.7\% versus $2.3 \%$ ).

Of those with any chronic condition, approximately $41 \%(n=972)$ reported having a single condition (physical or mental health), 23\% $(\mathrm{n}=548)$ reported having $2,15 \%(\mathrm{n}=344)$ reported 3 , and the remainder $(21 \% ; n=508)$ had 4 or more conditions. Among those reporting a single condition, approximately $19 \%(\mathrm{n}=181)$ reported having 1 single mental health condition. For those with 2 conditions, $68 \%(n=374)$ had 2 physical conditions, $9 \%(n=48)$ had 2 mental health conditions. 


\begin{tabular}{|c|c|c|c|}
\hline & Total sample & Males & Females \\
\hline \multicolumn{4}{|l|}{ Body System } \\
\hline \multicolumn{4}{|l|}{ Cardiovascular } \\
\hline heart attack in the last year & 1.3 & 1.7 & 0.9 \\
\hline congestive heart failure (CHF) & 3.5 & 4.0 & 3.1 \\
\hline angina or coronary artery disease & 4.4 & 6.0 & 2.9 \\
\hline other heart conditions, such as problems with heart valves or the rhythm of your heartbeat & 12.0 & 13.2 & 10.8 \\
\hline \multicolumn{4}{|l|}{ Endocrine } \\
\hline diabetes or high blood sugar & 15.0 & 16.9 & 13.1 \\
\hline \multicolumn{4}{|l|}{ Respiratory } \\
\hline chronic obstructive pulmonary disease (COPD) & 4.6 & 4.3 & 4.9 \\
\hline \multicolumn{4}{|l|}{ Musculoskeletal } \\
\hline rheumatoid arthritis (RA) & 8.2 & 7.9 & 8.4 \\
\hline osteoarthritis, degenerative arthritis & 15.0 & 10.9 & 19.1 \\
\hline osteoporosis & 6.6 & 2.3 & 10.7 \\
\hline \multicolumn{4}{|l|}{ Gastrointestinal } \\
\hline stomach disease, such as gastritis or duodenitis & 4.1 & 3.3 & 4.8 \\
\hline irritable bowel syndrome (IBS) & 8.3 & 5.7 & 10.8 \\
\hline kidney disease & 2.4 & 2.7 & 2.1 \\
\hline liver disease, such as Hepatitis B or C & 2.3 & 3.0 & 1.6 \\
\hline \multicolumn{4}{|l|}{ Other Physical Health } \\
\hline stroke & 3.2 & 3.4 & 3.0 \\
\hline migraine headaches & 14.9 & 7.5 & 21.9 \\
\hline chronic fatigue syndrome or fibromyalgia & 4.4 & 1.7 & 6.9 \\
\hline cancer, except skin cancer & 8.0 & 8.4 & 7.7 \\
\hline HIV or AIDS & 0.5 & 0.8 & 0.2 \\
\hline \multicolumn{4}{|l|}{ Mental Health } \\
\hline depression & 13.1 & 9.0 & 17.0 \\
\hline anxiety & 16.2 & 12.2 & 20.2 \\
\hline alcohol or other substance use disorder & 2.7 & 4.0 & 1.3 \\
\hline
\end{tabular}

Note: a) Hypertension, b) nasal allergies or allergic rhinitis, c) obesity, d) anemia, e) gastro-esophageal reflux disease (GERD), and f) sleep apnea, although included in the checklist, were excluded from analyses because they were not considered chronic conditions.

The remainder (23\%) reported having 1 physical and 1 mental health condition.

\section{HRQL burden of chronic physical health conditions}

Relative to participants in the Healthy group, those in the Physical Health Condition group showed substantial decrements in PCS scores. On average, males in the Physical Health Condition group scored 6.4 points lower (95\% Confidence Interval (CI) $[-7.3,-5.4]$ ) on PCS than did their Healthy counterparts. Similarly, females in the Physical Health Condition group scored 7.5 points lower (95\% CI $[-8.6,-6.4])$ than did females defined as Healthy. Relative to their PCS scores, decrements in MCS for the Physical Health Condition group were smaller for both males and females. Compared to scores for Healthy participants, MCS scores for males and females in the Physical Health Condition group were 1.7 points $(95 \%$ CI $[-2.7,-0.7])$ and 2.1 points $(95 \%$ CI $[-3.2,-0.9])$ lower, respectively. These results are shown in Table 3.

\section{HRQL burden of chronic mental health conditions}

Not surprisingly, the MCS scores for participants in the Mental Health Condition group revealed large decrements compared to scores for the Healthy group. Decrements were similar for males (11.3 points lower; $95 \%$ CI $[-13.3,-9.3])$ and females (11.9 points lower; 95\% CI $[-14.7,-9.0])$. For both males and females, PCS scores of those in the Mental Health Condition were not meaningfully different than PCS scores of those in the Healthy 
Table 3 Mean PCS and MCS decrements associated with a chronic physical health condition, a chronic mental health condition, or with co-morbid conditions (All compared to healthy reference group)

\begin{tabular}{|c|c|c|c|c|}
\hline & \multirow[b]{2}{*}{ Incremental decrement } & Males & \multirow[b]{2}{*}{ Incremental decrement } & \multirow{2}{*}{$\frac{\text { Females }}{\text { Total decremen }}$} \\
\hline & & Total decrement & & \\
\hline \multicolumn{5}{|l|}{ PCS } \\
\hline$\geq 1$ Chronic Physical Health Condition Only & $-6.4(-7.3,-5.4)$ & & $-7.5(-8.6,-6.4)$ & \\
\hline Addition of $\geq 1$ Chronic Mental Health Condition & $-4.5(-5.9,-3.2)$ & $-10.9(-12.3,9.6)$ & $-3.7(-4.9,-2.5)$ & $-11.2(-12.5,-9.9)$ \\
\hline$\geq 1$ Chronic Mental Health Condition Only & $-0.7(-2.6,1.2)$ & & $0.3(-2.5,3.1)$ & \\
\hline Addition of $\geq 1$ Chronic Physical Health Condition & $-10.2(-12.3,-8.1)$ & $-10.9(-12.3,9.6)$ & $-11.5(-14.3,-8.6)$ & $-11.2(-12.5,-9.9)$ \\
\hline \multicolumn{5}{|l|}{ MCS } \\
\hline$\geq 1$ Chronic Physical Health Condition Only & $-1.7(-2.7,-0.7)$ & & $-2.1(-3.2,-1.0)$ & \\
\hline Addition of $\geq 1$ Chronic Mental Health Condition & $-13.6(-15.0,-12.2)$ & $-15.3(-16.7,-14.0)$ & $-13.1(-14.3,-11.9)$ & $-15.2(-16.6,-14.1)$ \\
\hline$\geq 1$ Chronic Mental Health Condition Only & $-11.3(-13.3,-9.3)$ & & $-11.9(-14.7,-9.0)$ & \\
\hline Addition of $\geq 1$ Chronic Physical Health Condition & $-4.0(-6.2,-1.8)$ & $-15.3(-16.7,-14.0)$ & $-3.3(-6.2,-0.4)$ & $-15.2(-16.6,-14.1)$ \\
\hline
\end{tabular}

PCS = Physical Component Summary score.

MCS = Mental Component Summary score.

Values in parentheses are confidence intervals $(\mathrm{Cls})$.

group. Scores were 0.7 points lower $(95 \%$ CI $[-2.6,1.2])$ for males and 0.3 points higher $(95 \% \mathrm{CI}[-2.5,3.1])$ for females (Refer again toTable 3).

\section{Physical health conditions: incremental HRQL burden of mental health comorbidity}

Table 3 shows that for those in the Physical Health Condition group, a comorbid Mental Health Condition decreased PCS scores by an additional 4.5 points (95\% CI $[-5.9,-3.2])$ (males) and 3.7 points (95\% CI [-4.9, -2.5]) (females). Thus, total reductions were 10.9 and 11.2 points compared to the Healthy group. The incremental MCS burden of a mental health comorbidity to Physical Health Conditions alone was more pronounced, with increased decrements of -13.6 for males $(95 \% \mathrm{CI}=$ $[-15.0,-12.2])$ and -13.1 for females (95\% [-14.3, -11.9]). For both males and females, total MCS burden was approximately 15 points when the comorbid group was compared to the Healthy group.

\section{Mental health conditions: incremental HRQL burden of physical health comorbidity}

Finally, Table 3 shows that relative to participants in the Mental Health Condition group, those reporting both a Mental Health and a Physical Health Condition experienced lower HRQL as assessed by both PCS and MCS scores. PCS scores were 10.2 points lower (95\% [-12.3, -8.1]) for males and 11.5 points lower (95\% CI [-14.3, -8.6]) for females. Compared to those categorized as having a Mental Health Condition only, average additional decrements in MCS were 4.0 points $(95 \%$ CI $[-6.2,-1.8])$ and 3.3 points $(95 \%$ CI $[-6.2,-0.4)$ (respectively) for males and females.
HRQL burden across physical disease clusters

MCS decrements associated with a Mental Health Condition only (11.3 and 11.9 points, for males and females, respectively), along with the incremental effects for each of the 5 comorbid Physical Disease Clusters. Although comorbid gastrointestinal and musculoskeletal conditions were linked to the greatest overall MCS burden, the additional burden associated with a physical comorbidity was similar across each of the 5 clusters (3.6 to 5.6 points). Total MCS decrements were slightly larger for males than for females across the Physical Disease Clusters (data not shown).

Compared to the Healthy group, those in each Physical Disease Cluster showed PCS point reductions of 6.8 (gastrointestinal) to 11.7 (respiratory). Reductions were similar for males and females. The additional PCS reductions associated with a comorbid Mental Health Condition showed somewhat different patterns, depending on the particular Physical Disease Cluster. For 4 of 5 clusters, presence of a comorbid Mental Health Condition decreased PCS scores by approximately 5 additional points. For those with a cardiovascular condition however, a comorbid Mental Health Condition showed a smaller impact on PCS scores (-2.9 points). For each of the 5 Physical Disease Clusters, PCS decrements were almost identical for males and females (data not shown).

\section{Discussion}

The goal of this study was to examine the burden of self-reported chronic physical and/or mental health conditions on each of the two commonly-used SF36v2 summary measures of HRQL; the Mental Component 
Summary (MCS) score and the Physical Component Summary (PCS) score. Consistent with findings of prior studies, the impact of at least 1 chronic physical condition for this sample of adults in the US general population was reflected to a greater extent in PCS rather than MCS scores [20,39]. Similarly, the impact of at least 1 chronic mental health condition was primarily reflected in MCS rather than PCS.

Relative to individuals with only physical health conditions, males and females reporting $\geq 1$ comorbid mental health condition showed (on average) an additional PCS decrement of 4.5 and 3.7 points, respectively. These values are larger than 2-points, which is the recommended minimally important difference (MID) for group comparisons [35]. The finding that a comorbid chronic mental health condition was associated with an additional HRQL decrement is consistent with those reported in prior studies [15,17]. One of the strengths of the present study is that it provides quantitative information on specific PCS and MCS decrements associated with a comorbid condition related to mental health, as well as a comorbid condition related to physical health.

Compared to having a mental health condition only, the presence of $\geq 1$ comorbid physical health condition led to further MCS decrements of 3.3 points for females and 4.0 points for males. Both values are above the recommended MID for MCS, which is 3-points [35]. The observed incremental impact of a physical comorbidity on MCS and the incremental impact of mental health comorbidity on PCS scores were robust across groups organized by 'Physical Disease Clusters'. Total decrements in PCS scores associated with a chronic physical condition and a comorbid mental health condition were greater for the musculoskeletal, endocrine, and respiratory disease clusters (15.0 to 16.6 points) than for the gastrointestinal or cardiovascular disease clusters (11.7 to 12.0 points).

In the present study, overall patterns regarding HRQL decrements associated with physical and mental health comorbidities were similar for males and females. This finding differs from several other studies reporting lower HRQL scores for females with chronic physical or mental health conditions $[22,37,40,41]$.

Current findings underscore the complexity of identifying and managing patients with multiple chronic conditions, and the importance of screening for- and treating conditions related to both physical and mental health. To illustrate, results of a recent randomized clinical trial showed that for older adults with arthritis and comorbid depression, improvements in depressive symptoms were associated not only with decreased arthritisrelated pain, but with improved functional status and quality of life [7].

\section{Limitations}

As with all studies, current results should be interpreted within the context of relevant limitations. Our approach for this set of analyses was a broad-based one; with several types of data aggregation. These include the use of chronic disease groupings, disease clusters, and PCS and MCS Summary scores. While appropriate for assessing the incremental HRQL burden associated with chronic physical and/ or mental health conditions, certain distinctions were not assessed. These would include the 8 individual SF-36 domains, the different diseases within a particular disease cluster (e.g., type 1 versus type 2 diabetes), and different levels of disease severity. While beyond the scope of this study, future efforts may benefit from additional, more fine-grained analyses of the normative data.

The present results were based exclusively on selfreported information, with no diagnostic assessment or clinical records to provide corroboration. However, self report of chronic conditions has demonstrated reasonable levels of sensitivity and specificity when compared to information obtained from medical chart review [42,43]. Participants in the Healthy group may have had undiagnosed conditions or those not included in the chronic condition checklist. Finally, those with certain very severe or disabling chronic conditions, or those without internet access may have been precluded from participating in a panel survey, and therefore could be underrepresented.

Several factors potentially important in understanding the relationship of chronic conditions and HRQL were not included in the present set of analyses. For example, analyses did not incorporate bio-psychosocial risk factors such as smoking, being overweight and/or sedentary, or lacking social support; all of which have been shown to correlate with greater impairments in HRQL for those with chronic conditions $[21,24,30,44]$. To the extent allowed by participant sample sizes, future analyses might also include subgroups based on age, race/ethnicity, or income $[27,39,45]$. However, research has shown the magnitude of HRQL decrements associated with two or more chronic conditions to be largely independent of socioeconomic factors [27].

\section{Conclusions}

Results provide quantitative information for US adults on specific PCS and MCS score decrements associated with a comorbid condition related to mental health, as well as a comorbid condition related to physical health. Both PCS and MSC scores have shown to be significant predictors of outpatient and inpatient service utilization for individuals with chronic conditions such as arthritis. Thus, monitoring both aspects of functioning and wellbeing may lead to improved individual health outcomes and less use of costly healthcare services [46,47]. 
Note: A portion of this work was presented in poster format at the $16^{\text {th }}$ Annual International Meeting of the International Society for Pharmacoeconomics and Outcomes Research, May 21-25, Baltimore, MD.

\section{Competing interests}

At the time of original submission, all authors were full-time employees of QualityMetric, part of Optumlnsight Life Sciences, which publishes the SF$36 \mathrm{~V} 2^{\circledR}$ (the measure of HRQL for this paper).

\section{Authors' contributions}

All authors made substantive intellectual contributions to the study and/or the paper, and will take public responsibility for appropriate portions of its content. All have given final approval of the submitted version. MB: conception and design; revision of manuscript for important intellectual content. R R-B: conception and design; analysis and interpretation of data; revision of manuscript for important intellectual content. MKW: conception and design; revision of manuscript for important intellectual content. MM: draft of manuscript; revision of manuscript for important intellectual content. JB: conception and design; interpretation of data ST: draft of manuscript; revision of manuscript for important intellectual content. All authors read and approved the final manuscript.

Received: 12 July 2012 Accepted: 13 December 2012

Published: 19 December 2012

\section{References}

1. Centers for Disease Control and Prevention: Chronic Diseases and Health Promotion. 2010. Available at URL: http://www.cdc.gov/chronicdisease/ overview/.

2. National Center for Health Statistics: Health, United States, 2007. With Chartbook on Trends in the Health of Americans. Hyattsville, MD: National Center for Health Statistics; 2007. Available at URL: http://www.cdc.gov/ nchs/data/hus/hus07.pdf.

3. Anderson GF: Physician, public, and policymaker perspectives on chronic conditions. Arch Intern Med 2003, 163(4):437-42.

4. Center for Disease Control and Prevention (CDC): 2011 National Diabetes Fact Sheet. Diagnosed and Undiagnosed Diabetes in the United States.: All Ages; 2011. Available at URL: http://www.cdc.gov/diabetes/pubs/ estimates11.htm.

5. Mendes E: In US, chronic health conditions as prevalent in 2010 as 2009. 2011. Available at URL: http://www.gallup.com/poll/145868/chronic-healthconditions-prevalent-2010-2009.aspx?

6. Center for Disease Control and Prevention (CDC): Morbidity and Mortality Weekly. Prevalence of Coronary Heart Disease-United States, 2006-2010. 60th edition.: CDC; 2011:1377-81. Available at URL: http://www.cdc.gov/mmwr/ preview/mmwrhtml/mm6040al.htm?s_cid=mm6040al_w.

7. Lin EB, Katon W, Von Korff M, et al: Effect of improving depression care on pain and functional outcomes among older adults with arthritis. JAMA 2003, 290:2428-34

8. Kessler RC, Bergland P, Demler O, et al: Lifetime prevalence and age-ofonset distributions of DSM-IV disorders in the National Comorbidity Survey replication. Arch Gen Psychiatry 2005, 62:593-602.

9. Kessler RC, Chiu WT, Demler O, Walters EE: Prevalence, severity, and comorbidity of twelve-month DSM-IV disorders in the national Comorbidity Survey Replication (NCS-R). Arch Gen Psychiatry 2005, 62:617-27.

10. Chou K, Chi I: Prevalence of depression among elderly Chinese with diabetes. Int J Geriatr Psychiatry 2005, 20:570-5.

11. Müller-Tasch T, Frankenstein $L$, Holzapfel N, et al: Panic disorder in patients with chronic heart failure. J Psychosom Res 2008, 64:299-303.

12. Yawn B, Rocca LG, Wollan P: 10-Year trends in the diagnosis and treatment of hepatitis $C$ and concomitant mental health disorders: 1995 to 2005. Prim Care Companion J Clin Psychiatry 2008, 10:349-54

13. San Román AL, Muňoz F: Comorbidity in inflammatory bowel disease. World J Gastroenterol 2011, 17:2723-33.

14. Katon WJ: Clinical and health services relationships between major depression, depressive symptoms, and general medical illness. Biol Psychiaty 2003, 54:216-26.

15. Gadalla T: Association of comorbid mood disorders and chronic illness with disability and quality of life in Ontario Canada. Chronic Dis Can 2008, 28:148-54.
16. Chapman DP, Perry GS, Strine TW: The vital link between chronic disease and depressive disorders. Prev Chronic Dis 2005, available at URL: http:// www.cdc.gov/pcd/issues/2005/jan/04_0066.htm.

17. Das-Munshi J, Stewart R, Ismail K, et al: Diabetes, common mental disorders, and disability: findings from the UK National Psychiatric Morbidity Survey. Psychosomatic Med 2007, 69:543-50.

18. Prisciandaro JJ, Gebregziabher M, Grubaugh AL, et al: Impact of psychiatric comorbidity on mortality in veterans with type 2 diabetes. Diabetes Technol Therapeutics 2011, 13:73-8.

19. Fanuele JC, Birkmeyer NJ, Abdu WA, et al: The impact of spinal problems on the health status of patients: have we underestimated the effect? Spine 2000, 25(12):1509-14.

20. Mo F, Choi BCK, Li FCK, Merrick J: Using Health Utility Index (HUI) for measuring the impact on health-related quality of life (HRQL) among individuals with chronic diseases. The Scientific World 2004, 4:746-57.

21. Richardson LC, Wingo PA, Zack MM, et al: Health-related quality of life in cancer survivors between ages 20 and 64 years: population-based estimates from the Behavioral Risk Factor Surveillance System. Cancer 2008, 112:1380-9.

22. Hopman WM, Harrison MB, Coo H, et al: Associations between chronic disease, age and physical and mental health status. Chronic Dis Can 2009, 29:108-16.

23. Cook EL, Harman JS: A comparison of health-related quality of life for individuals with mental health disorders and common chronic medical conditions. Public Health Rep 2008, 123:45-51.

24. Chen H-Y, Baumgardner DJ, Rice JP: Health-related quality of life among adults with multiple chronic conditions in the United States, Behavioral Risk Factor Surveillance System, 2007. Prev Chronic Dis 2011, 8:A09. Available at URL: http://www.cdc.gov/pcd/issues/2011/jan/09_0234.htm.

25. Moriarty DG, Zack MM, Kobau R: The centers for disease control and prevention's healthy days measures-population tracking of perceived physical and mental health over time. Health Qual Life Outcomes 2003, 1(37).

26. Centers for Disease Control and Prevention: Measuring healthy days: population assessment of health-related quality of life. Atlanta GA: CDC; 2000. http://www.cdc.gov/HRQL/pdfs/mhd.pdf.

27. Rothrock NE, Hays RD, Spritzer $K$, et al: Relative to the general US population, chronic diseases are associated with poorer health-related quality of life as measured by the Patient-Reported Outcomes Measurement System (PROMIS). J Clin Epidemiol 2010, 63:1195-1204.

28. Centers for Disease Control and Prevention: Behavioral Risk Factor Surveillance System survey data. 2007. http://www.cdc.gov/brfss/ technical infodata/surveydata/2007.htm.

29. Fortin M, Dubois MF, Hudon C, et al: Multimorbidity and quality of life: a closer look. Health Qual Life Outcomes 2007, 5(52).

30. Fortin M, Lapopint L, Hudon C, et al: Multimorbidity and health-related quality of life in primary care: a systematic review. Health Qual Life Outcomes 2004, 2(51).

31. Alonso J, Ferrer M, Gandek B, et al: Health-related quality of life associated with chronic conditions in eight countries: results from the International Quality of Life Assessment (IQOLA) Project. Qual Life Res 2004, 13:283-98.

32. Bayliss EA, Bayliss MS, Ware JE Jr, Steiner JF: Predicting declines in physical function in persons with multiple chronic medical conditions: what we can learn from the medical problems list. Health Qual Life Outcomes 2004, 7:47.

33. Ware JE, Sherbourne CD: The MOS 36-item short form health survey (SF-36). I. Conceptual framework and item selection. Med Care 1992 30:473-83

34. Ware JE Jr, Kosinski M, Bjorner JB, et al: User's Manual for the SF-36v2 ${ }^{\circledR}$ Health Survey. 2nd edition. Lincoln, Rl: QualityMetric Inc.; 2007.

35. Maruish ME (Ed): User's Manual for the SF-36v2 ${ }^{\circledR}$ Health Survey. 3rd edition. Lincoln, Rl: QualityMetric Inc.; 2011.

36. KnowledgePanel ${ }^{\circledR}$ Design Summary: Knowledge Networks 2010. http://www. knowledgenetworks.com/ganp/index.html.

37. Sprangers MA, de Regt EB, Andries F, et al: Which chronic conditions are associated with better or poorer quality of life? J Clin Epidemiol 2000 53:895-907.

38. Varni JW, Limbers CA, Burwinkle TM: Impaired health-related quality of life in children and adolescents with chronic conditions: a comparative analysis of 10 disease clusters and 33 disease categories/severities utilizing the PedsQL 4.0 Generic Core Scales. Health Qual Life Outcomes 2007. 
39. Wikman A, Wardle J, Steptoe A: Quality of life and affective well-being in middle-aged and older people with chronic medical illnesses: a crosssectional population based study. PLoS One 2011, 6:e18952. Available at URL: http://www.plosone.org.

40. Ronen D, Mor A, Segal O, et al: Differences in gait patterns, pain, function and quality of life between males and females with knee osteoarthritis: a clinical trial. BMC Musculoskelet Disord 2009, 10:127.

41. Gray LJ, Sprigg N, Bath PMW, et al: Sex differences in quality of like in stroke survivors: data from the Tinzaparin in Acute Ischaemic Stroke Trial (TAIST). Stroke 2007, 38:2960-4

42. Martin LM, Leff M, Calonge N, et al: Validation of self-reported chronic conditions and health services in a managed care population. Am J Prev Med 2000, 18:215-8.

43. Bayliss EA, Ellis JL, Steiner JF: Subjective assessments of comorbidity correlate with quality of life health outcomes: Initial validation of a comorbidity assessment instrument. Health Qual Life Outcomes 2005, 3:51.

44. Strine TW, Chapman DP, Balluz L, Mokdad AH: Health-related quality of life and health behaviors by social and emotional support. Their relevance to psychiatry and medicine. Soc Psychiatry Psychiatr Epidemiol 2008, 43:151-9.

45. Cutshaw CA, Staten LK, Reinschmidt KM, et al: Depressive symptoms and health-related quality of life among participants in the Pasos Adelante Chronic Disease Prevention and Control Program, Arizona, 2005-2008. Prev Chronic Dis 2012, Available at URL: http://www.cdc.gov/pcd/issues/ 2012/.

46. Singh JA, Nelson DB, Fink HA, Nichol KL: Health-related quality of life predicts future health care utilization and mortality in veterans with selfreported physician-diagnosed arthritis: The Veterans' Arthritis Quality of Life Study. Semin Arthritis Rheum 2004, 34:755-65.

47. Singh JA, Borowsky SJ, Nugent S, et al: Health-related quality of life, functional impairment, and healthcare utilization by veterans: Veterans' Quality of Life Study. J Am Geriatr Soc 2005, 53:108-13.

doi:10.1186/1477-7525-10-154

Cite this article as: Bayliss et al: Health-related quality of life (HRQL) for individuals with self-reported chronic physical and/or mental health conditions: panel survey of an adult sample in the United States. Health and Quality of Life Outcomes 2012 10:154.

\section{Submit your next manuscript to BioMed Central and take full advantage of:}

- Convenient online submission

- Thorough peer review

- No space constraints or color figure charges

- Immediate publication on acceptance

- Inclusion in PubMed, CAS, Scopus and Google Scholar

- Research which is freely available for redistribution 